\title{
Upstream paths for Hippo signaling in Drosophila organ development
}

\author{
Kwang-Wook Choi* \\ Department of Biological Sciences, Korea Advanced Institute of Science and Technology, Daejeon 34141, Korea
}

\begin{abstract}
Organ growth is fundamental to animal development. One of major mechanisms for growth control is mediated by the conserved Hippo signaling pathway initially identified in Drosophila. The core of this pathway in Drosophila consists of a cascade of protein kinases Hippo and Warts that negatively regulate transcriptional coactivator Yorkie (Yki). Activation of Yki promotes cell survival and proliferation to induce organ growth. A key issue in Hippo signaling is to understand how core kinase cascade is activated. Activation of Hippo kinase cascade is regulated in the upstream by at least two transmembrane proteins Crumbs and Fat that act in parallel. These membrane proteins interact with additional factors such as FERM-domain proteins Expanded and Merlin to modulate subcellular localization and function of the Hippo kinase cascade. Hippo signaling is also influenced by cytoskeletal networks and cell tension in epithelia of developing organs. These upstream events in the regulation of Hippo signaling are only partially understood. This review focuses on our current understanding of some upstream processes involved in Hippo signaling in developing Drosophila organs. [BMB Reports 2018; 51(3): 134-142]
\end{abstract}

\section{INTRODUCTION}

Genetic regulation of cell proliferation and survival is crucial for normal patterning and growth of organs. Drosophila melanogaster has been an ideal model animal to dissect the molecular genetic basis of development. Major signaling pathways including Wingless/Wnt, Notch, and Hedgehog signaling discovered in Drosophila have provided insights necessary to uncover conserved mechanisms in mammalian development and understand the molecular basis of related human diseases (1).

*Corresponding author. Tel: +82-42-350-2644; Fax: +82-42-3505644; E-mail: kchoi100@kaist.ac.kr

https://doi.org/10.5483/BMBRep.2018.51.3.027

Received 28 December 2017

Keywords: Cytoskeleton, Drosophila, Growth control, Hippo signaling, Organ development
In studying organ development, the eye and the wing have been extensively used as model organs. Adult eyes and wings derive from eye and wing imaginal discs, respectively. Imaginal discs are relatively simple epithelial organ primordia. During larval stages, imaginal discs are specified as different organ precursors. They develop by cell proliferation and patterning processes. Genetic defects causing overgrowth or hypotrophy of these organs can be easily detected in larvae or adult flies. Eyes and wings are dispensable for animal's survival, making these organs particularly attractive for genetic analysis of organ development.

Genetic screens using unbiased mutagenesis have been powerful for discovering Hippo pathway mutants. Genetic mosaic techniques have been utilized to search for mutations that affect tissue growth in vivo. Mitotic recombination can be induced to generate genetic mosaic tissues in which wild-type cells and mutant sister cells can be visualized. The lats tumor suppressor gene was first identified by a mutation that caused tumorous growth in mitotic clones (2). The same gene named warts (wts) was also found by an independent clonal analysis (3). More recently, a modified genetic strategy has been used to isolate mutations affecting tissue growth. In this approach, mutant clones in the eye are marked by white gene mutation. By comparing the size of white mutant clones and red wild-type sister clones, one can identify mutations that affect tissue growth in the eye. From this screen, several mutant alleles for another Hippo pathway gene salvador (sav) have been isolated (4). In addition, several groups have identified hippo mutations that cause massive overgrowth in eye and wing organs (5-8). Identification of Wts, Sav, and Hpo from these studies has laid a foundation for the Hippo signaling pathway that regulates organ growth.

Since the identification of these core factors, there have been expanded investigations in Drosophila and mammalian systems to refine the pathway with a number of newly discovered components (9-12) (Table 1). Hippo signaling in mammalian systems and human diseases has been covered by other reviews in this issue. This review begins with a brief overview of the Hippo pathway. It mainly focuses on upstream components and roles of cytoskeleton in Hippo signaling during Drosophila organ development.

ISSN: 1976-670X (electronic edition)

Copyright (C) 2018 by the The Korean Society for Biochemistry and Molecular Biology

(c) This is an open-access article distributed under the terms of the Creative Commons Attribution Non-Commercial License (http://creativecommons.org/licenses/by-nc/4.0) which permits unrestricted non-commercial use, distribution, and reproduction in any medium, provided the original work is properly cited. 
Table 1. Members of the Hippo signaling pathway in Drosophila and human

\begin{tabular}{llll}
\hline \multicolumn{1}{c}{ Function } & Drosophila & \multicolumn{1}{c}{ Human } & Protein family \\
\hline \multirow{2}{*}{ Transmembrane signaling } & Fat (Ft) & Ft1-4 & Atypical cadherin \\
& Dachsous (Ds) & DCHS1,2 & Atypical cadherin \\
& Dachs (D) & $?$ & Atypical myosin \\
& Crumbs (Crb) & CRUMBS1-3 & Transmembrane protein \\
FERM protein complex & Expanded (Ex) & FRMD1,6 & FERM domain protein \\
& Merlin (Mer) & MERLIN/NF2 & FERM domain protein \\
& Kibra (Kibra) & KIBRA & WW domain protein \\
Kinase cascade & Schip1 (Schip1) & SCHIP1 & Ex/Merlin-interacting protein \\
& Tao-1 (Tao-1) & TAO1-3 & Ste20 family protein kinase \\
& Hippo (Hpo) & MST1,2 & Ste20 family protein kinase \\
& Salvador (Sav) & SAV1/WW45 & Scaffolding protein \\
Transcription & Warts (Wts) & NATS1,2 & NDR family protein kinase \\
& Yorkie (Yki) & YAP, TAZ & Transcription coactivator \\
Actin regulators & Scalloped (Sd) & TEAD1-4 & Transcription factor \\
& Cpa & CAPZA1-3 & Actin capping protein \\
& Cpb & CAPZB & Actin capping protein \\
& Twin star (Tsr) & COFILIN & Actin depolymerization factor \\
& Ajuba & LIMD1, WTIP, AJUBA & LIM domain protein \\
\hline
\end{tabular}

\section{HIPPO-WARTS KINASE CASCADE AT THE CENTER}

Wts, Sav, and Hpo share function as tumor suppressors for negative growth regulation $(5,8)$. Genetic analysis of their relationships in developing organs has revealed that they act together to form a core in a pathway (Fig. 1). Hpo and Wts are serine/threonine protein kinases that belong to STE-20 and NDR family kinases, respectively $(2,3,13)$. Hpo directly activates Wts by phosphorylation. Wts can also be phosphorylated by an alternative Hpo-like kinase Happyhour (14).

In the search for Wts-interacting proteins, a transcriptional coactivator named Yorkie (Yki) after the Yorkshire Terrier has been identified as a regulator that controls both cell proliferation and survival (15). Yki is required for transcriptional activation of Cyclin E (CycE) and Death-associated inhibitor of apoptosis 1 (Diap1) that promotes cell cycle and inhibits cell death, respectively (7). Yki also regulates the expression of bantam microRNA to increase cell proliferation and survival $(16,17)$. Scalloped $(S d)$ mutations cause characteristic wing notching phenotypes (18). Sd is a TEA domain transcription factor that forms a dimer with Vestigial $(\mathrm{Vg})$ to induce wing development $(19,20)$. Yki coactivator binds to Sd to activate CycE and Diap1 gene transcription (21-23). Unlike Yki, Sd is not required for all imaginal discs, suggesting that Yki might interact with different transcription factors to induce tissue-specific growth and cell survival. Although Sd is not required for normal eye development, it is critical for compensatory proliferation when cell death is induced in developing eye disc (24).

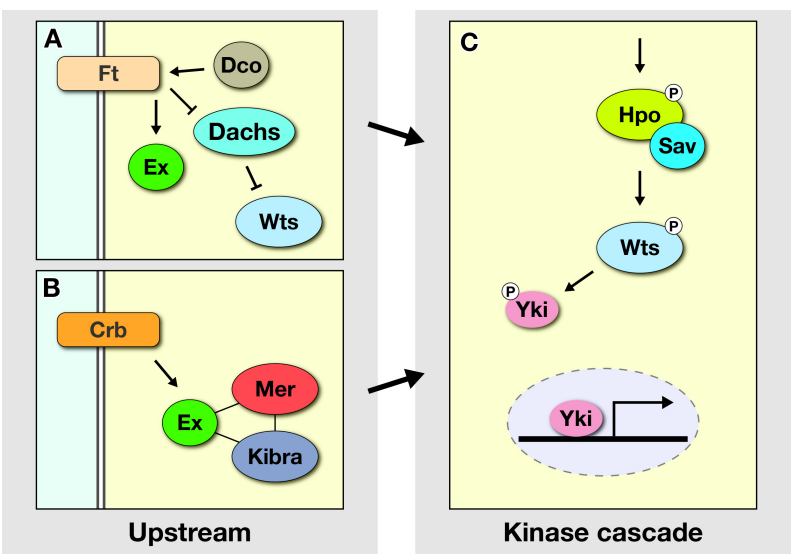

Fig. 1. Upstream pathways for Hippo signaling. Hippo signaling is regulated by two upstream events. (A) Transmembrane protein Ft activates Wts by inhibiting Dachs. Dco promotes Ft activity by phosphorylation. Ft also regulates Ex localization. (B) Crb binds to Ex. Ex, Mer, and Kibra function together to activate the Hippo kinase cascade. (C) The core kinase cascade. Hpo, together with Sav, activates Wts through phosphorylation. Activated Wts phosphorylates Yki to block its nuclear entry.

\section{FAT AND CRUMBS AT THE CELL MEMBRANE}

Cell-cell communication is essential for controlled growth of epithelial cells during organ development. Growth signaling is mediated by membrane-bound proteins located at cell junctions. Two transmembrane proteins, Fat (Ft) and Crumbs 
(Crb), have been identified as important upstream factors that lead to the activation of the Hippo kinase cascade (Fig. 1A, B).

$\mathrm{Ft}$ and Dachsous (Ds) are atypical cadherin proteins involved in the regulation of planar cell polarity in developing eye and wing epithelia (25). In Hippo signaling, Ds activates Ft by binding to $\mathrm{Ft}$ as a membrane-bound ligand followed by a relay of $\mathrm{Ft}$ signal for $\mathrm{Hpo}$ activation. Loss of $\mathrm{Ft}$ causes overproliferation which results in enlarged organs. Ft activates Hippo signaling in two ways. Firstly, Ft is necessary for the localization of a FERM domain protein Expanded (Ex) at apical junctions to promote Hpo signaling (26-30). Loss of Ft affects the stability of Ex without altering the localization or level of $\mathrm{Hpo}$, suggesting that Ft affects Hpo phosphorylation mainly by regulating the localization of Ex, but not $\mathrm{Hpo}$ (26). Secondly, Ft inhibits apical localization of atypical myosin Dachs. Dachs promotes growth by inhibiting Wts function (31). An $\mathrm{SH} 3$ domain protein Vamana can directly interact with Dachs. Such interaction mutually facilitates their apical localization (32). Ft activity is promoted by phosphorylation in the intracellular domain through a Casein kinase $1 \varepsilon$ Disc-overgrown (Dco) (33). Effects of Dco can be antagonized by palmitoylation of Ft by palmitoyltransferase Approximated (App). Thus, Ft activity and apical Dachs localization are regulated by two opposing posttranslational modifications (34).

$\mathrm{Crb}$ is another transmembrane protein that acts upstream of the Hippo pathway in parallel with Ft branch (Fig. 1B, C). Crb is well known for its critical function in the establishment and maintenance of apical basal epithelial cell polarity (35). Evidence suggests that Crb function is regulated by homotypic extracellular binding (36). Crb acts upstream of the Hippo kinase cascade for growth regulation (37-40). Mammalian Crb3, a major isoform expressed in epithelial cells, is also involved in the regulation of Hippo-YAP pathway (41). The intracellular domain of $\mathrm{Crb}$ ( $\left.\mathrm{Crb}^{\text {intra }}\right)$ has two functional motifs: the juxtamembrane FERM-binding motif (FBM) and the PDZ binding motif (PBM). Of these motifs, the FBM domain is essential for Crb function in Hippo signaling by binding to the FERM domain of Ex. Mutation in FBM leads to mislocalization of the apical Ex protein to the basal region of cells in imaginal discs. Thus, Crb is necessary for recruiting Ex to the apical plasma membrane.

Overexpression of full-length $\mathrm{Crb}$ or $\mathrm{Crb}^{\text {intra }}$ results in overgrowth of imaginal discs, similar to the effect of Crb loss-of-function (LOF). An explanation for this phenomenon has been provided. In the absence of Crb, Hippo signaling is inactivated because Ex cannot be recruited to Crb. Interestingly, high levels of $\mathrm{Crb}$ by overexpression promote phosphorylation of Ex which causes proteasome-dependent degradation of Ex by Skp-Cullin-F-Box (SCF) E3 ubiquitin ligase complex (42). Hence, overexpression of Crb can also inhibit Hippo signaling by reducing Ex level. F-box protein Slimb (Slmb) is responsible for Ex degradation induced by high levels of Crb. However, protein kinases necessary for Ex phosphorylation have not been identified.
Collectively, Ft and Crb form major upstream pathways to activate the Hippo kinase cascade. Dachs is recruited to apical membranes by interacting with Ft whereas Ex is localized by both $\mathrm{Crb}$ and $\mathrm{Ft}$. Crb-induced degradation of Ex provides a mechanism for controlled growth in response to changes in Crb level.

\section{EX-MER-KIBRA FOR RELAY}

In parallel with Ex, Merlin (Mer), another FERM-domain protein, is required for mediating the function of Crb. Drosophila Mer is the homolog of mammalian Mer (Schwanommin), a tumor suppressor of neurofibromatosis type 2 (NF2). Mutations in NF2 are associated with various neural diseases (43). Drosophila Mer and ex are structurally related. They also show genetic interaction. Mutations in either Mer or ex show mild defects in wing growth. Double mutations in both genes result in strong synergistic effects on tumorous tissue growth (44-46). Thus, Mer and Ex can act in partially redundant manner for Hpo signaling. Genetic studies have revealed that Mer and Ex act upstream of the Hpo-Wts pathway to negatively regulate Yki activity, consistent with tumor suppressor function of NF2 (47).

Kibra is another protein that acts upstream of Hpo-Wts core kinases. Human KIBRA was initially found as a WW domain protein enriched in the kidney and the brain (48). Drosophila kibra was identified in a genetic screen for cell polarity factors in the oocyte. Kibra physically interacts with Mer and Ex to form a protein complex localized to the apical cell membrane (49) (Fig. 1). Kibra can enhance the level of Wts phosphorylation induced by Mer and Ex in vitro, consistent with synergistic tissue overgrowth by mutations in kibra and ex (or Mer). Furthermore, Kibra, Mer, and Ex can directly bind to the Hpo-Sav complex, thus activating Wts kinase activity $(50,51)$. Kibra also interacts with Wts. Interestingly, when Yki is not phosphorylated by Wts, Ex can directly sequester Yki (52). Because depletion of Ex or Kibra does not affect the interaction between Wts and Yki, Ex and Kibra seem to be mainly required to activate the Hippo kinase cascade rather than promote Wts-Yki interaction (51). Depletion of Kibra also reduces the strength of Ex-Mer interaction, indicating a role of Kibra in stabilizing the Ex-Mer complex (51).

Although Ex, Merlin, and Kibra function together to activate the Hippo pathway, each of these factors may have tissue-specific contribution in vivo. Effects of ex mutations are more severe in imaginal discs than those of Mer mutations. On the contrary, Mer is essential for regulation of cell polarity and growth suppression in ovarian follicle cells (53). Ex and Mer also display differential roles in regulating cell cycle and apoptosis in different tissues and organs (47). Thus, Ex, Mer, and Kibra proteins might form distinct protein complexes with tissue-specific factors for context-dependent regulation of Hippo signaling.

It is interesting to note that Kibra and Mer are not only 
detected at the junctional domain of wing disc epithelial cells, but also detected in the apical medial region between junctions $(54,55)$ (Fig. 2). Depletion of Kibra and Mer leads to strong reduction of Sav at the medial domain, but not at junctions. Kibra and Mer also recruit Hpo to the medial region for Sav-dependent activation of Hpo signaling. In contrast to Mer, Ex is only localized to the junctional membrane, but not to the medial region. Junctional localization of Kibra requires neither Mer nor Ex. Instead, Crb plays a key role in sequestering Kibra to apical junctions, consistent with medial accumulation of Kibra in crb mutant clones. This leads to a proposal that Hippo signaling is organized at two distinct sites of the apical membranes: junctional and medial regions. Thus, $\mathrm{Crb}$ has dual functions to suppress $\mathrm{Hpo}$ signaling by degrading Ex and sequestering Kibra away from the medial domain. It remains to be determined whether the apical medial localization of Kibra is a general phenomenon in the epithelia of other organs.

\section{SCHIP1 AND TAO-1 BRIDGE EX TO THE HPO CASCADE}

Ex, Mer, and Kibra form a protein complex with Hpo to activate the kinase cascade. However, they are not directly linked to Hpo kinase, suggesting that additional factors are needed to connect Ex-Mer-Kibra to Hpo. Two independent studies have identified Tao-1 kinase as a direct regulator of Hpo $(56,57)$. Human TAO1 can suppress the activity of YAP in human cells, suggesting that human TAO kinases might function as tumor suppressors (57).

Tao-1 is a serine/threonine protein kinase that promotes Hpo activation by phosphorylation. Genetic evidence indicates that Tao-1 acts downstream of the Ex-Mer-Kibra complex. However, given that Ex-Mer-Kibra does not directly interact with Tao-1 (56), there might be additional protein(s) that can bind to the Ex-Mer-Kibra complex. Drosophila Schip1 (hereafter Schip1), a homolog of human Schwanommin (Merlin) interacting protein 1 (hSchip1) (55), turns out to be a factor that connects Ex-Mer and Tao-1 (58). Loss of Schip1 increases Yki activity and induces organ overgrowth (58). Schip1 is recruited apically to Ex by direct binding. Furthermore, Schip1 directly promotes Tao-1 activity by physical interaction, leading to increased phosphorylation of Hpo. Therefore, Schip1 and Tao-1 play critical roles in connecting Ex to Hpo, hence facilitating the activity of the Hpo kinase cascade (Fig. 2).

Tao-1 was initially found as a protein that could control cell shape by destabilizing the microtubule plus-end (59). A recent study has revealed new functions of Tao- 1 in germline cell development (60). Interestingly, alternative promoters of tao-1 produce two different proteins (Tao-L and Tao-S) that act antagonistically. The long form Tao-L, but not the short form Tao-S, contains the protein kinase domain in the $\mathrm{N}$ terminal region. Tao-L and Tao-S can have distinct effects on cell shape by inducing lamellapodia and filopodia, respectively. Thus, Tao-1 can affect both microtubule and actin cytoskeletons by Tao-L and Tao-S.

The function of Tao-1 in organ growth depends on its kinase activity (56). Therefore, it is likely that Tao-L plays a major role

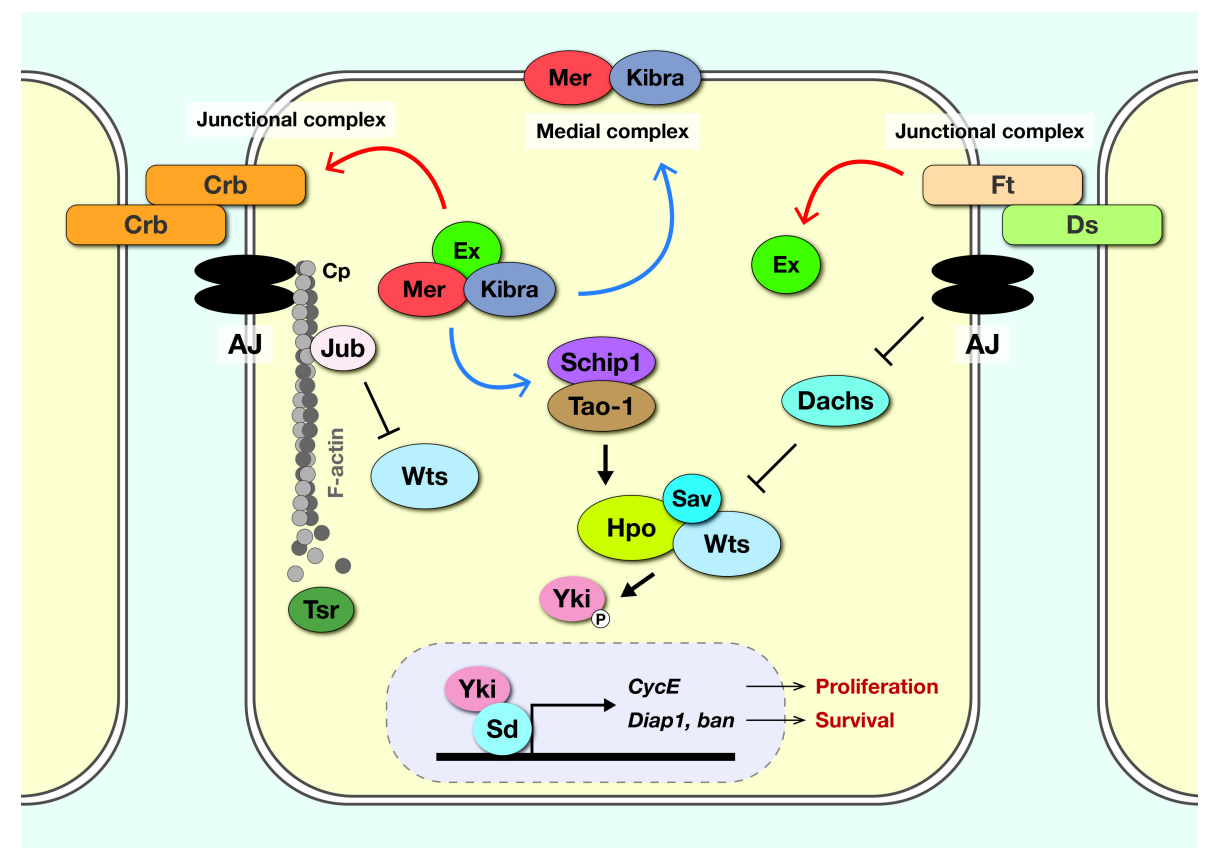

Fig. 2. Overview of the Hippo signaling pathway in Drosophila. Key steps of Hippo signaling. (1) Membrane proteins $\mathrm{Ft}$ and $\mathrm{Crb}$ regulate junctional localization of Dachs and Ex-Mer-Kibra, respectively. (2) Ex-Mer-Kibra function together to activate the Hippo kinase cascade. Mer and Kibra can form a complex at cell junction (Junctional complex) and at apical medial region (Medial complex). Ex binds to Schip1 which then promotes Tao-1 kinase activity for Hpo phosphorylation. (3) Hpo-Wts core kinase cascade. Activated Wts phosphorylates Yki to block its nuclear entry. F-actin formation and $\mathrm{Yki}$ activation are negatively regulated by capping proteins (Cp) and Tsr/cofilin. (4) When Wts is inactive, Yki induces target gene expression to promote cell proliferation and cell survival. 
in activating Hpo for growth suppression. However, it is unknown whether both Tao-L and Tao-S are expressed in imaginal discs to affect microtubule and actin cytoskeletons or whether they act in an antagonistic manner. Tao-1 kinase is required for the restriction of larval brain size by regulating neuroblast proliferation through Hippo signaling (61). Reduction of Mer causes enlargement of the adult head that can be suppressed by Schip1 overexpresssion $(58,62)$. It needs to be determined whether effects of Mer and Schip1 on head size depends on Tao-1 function. Like Tao-1, Mer is also associated with microtubules. Recent studies have shown that Hippo components are required for microtubule polarity, axial patterning, and asymmetric cell division in developing tissues (63-65). Hence, functional relationship between microtubules and Hippo signaling is an intriguing topic to be explored in the future.

\section{ACTIN CYTOSKELETON IN HIPPO SIGNALING}

Crb is essential for epithelial integrity by regulating adherens junctions (AJs). The FERM domain of Ex can bind to the FBM region of the intracellular domain of $\mathrm{Crb}(39,40)$. This raises the possibility that actin cytoskeleton associated with the FERM domain might be involved in the regulation of Hippo signaling.

Multiple proteins involved in actin dynamics have been identified as regulators of the Hippo signaling pathway in Drosophila. Loss of actin capping protein alpha (Cpa) and beta (Cpb) or overexpression of an active form of a formin family protein Diaphanous induces F-actin formation and overgrowth in imaginal discs (66). F-actin-induced overgrowth depends on Yki activity. Importantly, tissues overgrown by excess F-actin show relatively normal cell polarity, suggesting that F-actin accumulation has direct effects on tissue growth by modulating the Hpo pathway rather than through disrupting epithelial cell polarity. The role of F-actin in Hippo signaling seems to be evolutionarily conserved based on the findings that increased F-actin assembly or mDia overexpression causes activation of mammalian Yap activity (66).

Independent studies have found similar roles of actin dynamics. $c p b$ mutant clones in wing discs show overgrowth without causing any defects in cell polarity (67). Interestingly, clone boundaries are smooth and round, suggesting that mutant clone cells and adjacent wild-type cells have different tension and affinity properties. This seems to be consistent with previous observation showing that junctional tension is modulated by loss of tumor suppressors in mutant clones (68). Capulet (Capt) is a cyclase-associated protein that inhibits actin polymerization by sequestering actin monomers. Knockdown of Capt results in F-actin accumulation and increases Yki target gene expression. Drosophila cofilin encoded by twin star (tsr) severs actin filaments by depolymerizing actin from the pointed end of F-actin. Knockdown of Tsr by RNAi potently increases the Yki transcriptional activity in cell culture assay
(66). Clones of weak tsr mutant alleles in imaginal discs show increased F-actin accumulation as in $c p b$ mutant clones without inducing overgrowth or activation of Yki target genes. This suggests that accumulation of F-actin is insufficient to induce Yki activation (67). However, clones generated by stronger $t s r$ alleles display F-actin accumulation and upregulation of ex-lacZ reporter, suggesting Yki activation (69). Cells mutated in $t s r$ or cpa show basal extrusion from the epithelia, suggesting that both genes are required for the maintenance of epithelial integrity. Despite similarities between effects of cpa and $t s r$ mutations, they have apparent differences. In contrast to increase in Arm level of cpb LOF mutant clones, reduced Tsr causes strong reduction of Arm and extensive cell death. Hence, Tsr not only restricts cell proliferation, but also promotes cell survival, consistent with the observation that $t s r$ RNAi induces overproliferation when cell death is blocked by expressing baculovirus cell death inhibitor gene p35 (69). In contrast, although Cpa is required for cell survival, dying cells are not rescued by p35 overexpression, indicating that dying cells in cpa mutants are independent of programmed cell death (70).

Mechanisms underlying tissue overgrowth triggered by misregulation of F-actin have not been clearly identified. Reduction of Cpa does not affect subcellular localization of Ex, Mer, or Hpo, but increases nuclear localization of Yki. Phenotypes of ex mutants can be rescued by Hpo overexpression, but not by active Dia, suggesting that F-actin acts in parallel with Ex and Hippo to regulate the localization of Yki (66). Loss of Ex, but not Mer, reduces the level of F-actin in the wing disc (67), supporting that Ex regulates actin cytoskeleton in vivo by binding to F-actin. On the other hand, clonal loss of hpo, sav,

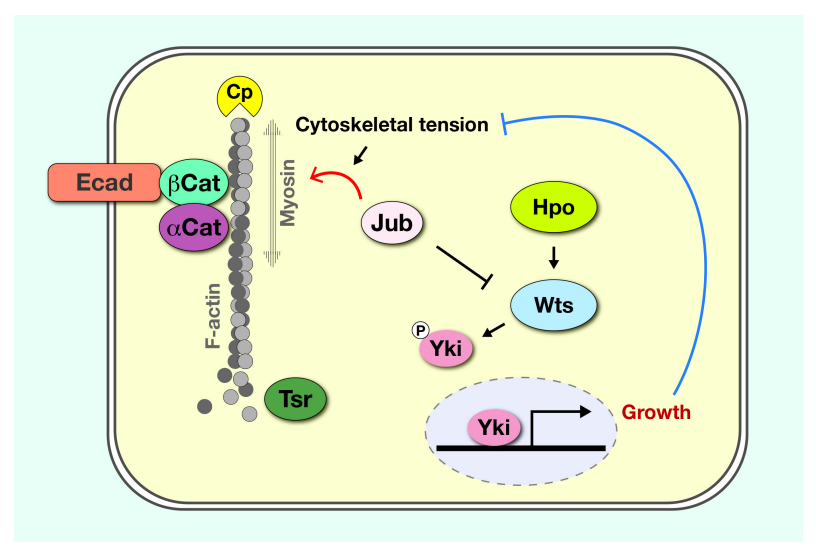

Fig. 3. Role of actin cytoskeleton in Hippo signaling. Cell adhesion is regulated by Ecad, $\beta$-catenin ( $\beta$ Cat), and $\alpha$-Catenin ( $\alpha$ Cat) at the adherens junction. $\alpha$ (Cat) interacts with actomyosin networks. Under cell tension, Jub is apically localized with Wts to inactivate Wts, increasing Yki activity for growth. Fast growth reduces cytoskeletal tension by feedback to decrease junctional localization of Jub and Wts, thus decreasing Yki activity. 
or wts induces F-actin accumulation, implying the presence of negative feedback regulation between F-actin and the Hpo kinase cascade activity (67). How Hpo pathway activity regulates F-actin and vice versa are intriguing issues to be studied further.

Cells in developing tissues are influenced by extracellular environments including neighboring cells. Cells respond to environmental changes by sensing mechanical forces and transmitting surface tension signals into cells. Mechanical conditions of a tissue can affect the progression of diseases. Loss of tensional homeostasis can enhance cancer risk (71). Recent studies in Drosophila and mammals have provided strong evidence that cell tension affects organ growth by modulating Hippo signaling (72-74). In this process, actin cytoskeleton and its regulators play key roles in mediating cell tension signals. In the wing disc, a LIM domain protein Ajuba (Jub) plays a role in relation to cytoskeletal tension and tissue growth (75-79). $\alpha$-Catenin, a key factor for mechanosensing and adherens junction development $(80,81)$, is required for localizing Jub to junctions (Fig. 3). Jub is increased at compartment boundaries that are sites of increased tension between two different groups of cells. Reducing tension by depleting Rho-associated kinase (ROCK) encoded by rho kinase gene decreases the level of apical Jub. Conversely, enhancing tension by overexpression of ROCK or an active form of the regulatory light chain of Myosin II encoded by spaghetti squash (sqh) increases apical Jub (77). Apical Jub recruits Wts to AJs, thereby inhibiting Wts function in a tension-dependent manner.

Zyxin (Zyx) is another LIM-domain protein associated with actin. Zyx is recruited to actin compromised by mechanical stress. It repairs actin fibers by recruiting actin polymerase Enabled (Ena) (82). This interaction is mediated by the L/FPPPP motif of Zyx (83). RNAi-based studies have suggested that Zyx functions downstream of Ft independent of Ex (84). However, analysis using Zyx null mutants has provided evidence that Zyx antagonizes Ex (83). Although Zyx null mutants are viable and fertile, they display smaller body sizes than normal ones. Zyx LOF phenotypes are suppressed by ex LOF mutation, but not by ft LOF mutation. Phenotypes of Ex overexpression can also be suppressed by Zyx overexpression, supporting that Zyx antagonizes Ex (85). These studies have led to a model that Zyxin can promote tissue growth by binding to Ena, thus facilitating F-actin polymerization. On the other hand, Ex and $\mathrm{Cpa} / \mathrm{Cpb}$ capping proteins inhibit tissue growth by preventing actin polymerization. Thus, the antagonistic relationship between Zyx and Ex on Yki activation appears to be mediated through their opposing effects on F-actin polymerization (83). In contrast to the role of Jub in Wts inhibition, function of Zyx in promoting $\mathrm{Yki}$ activation is independent of Wts. This seems to be consistent with mammalians studies showing that YAP regulation by mechanical stretching in mammalian cells is independent of LATS $(72,86,87)$.

Spectrin is part of the cytoskeleton that supports the integrity of cell structures. Drosophila encodes one $\alpha$ subunit ( $\alpha$-Spec) and two $\beta$ subunits ( $\beta_{\text {Heavy }}$-Spec or $\beta$-Spec) that generate two heterodimers: $\alpha \beta$ and $\alpha \beta_{\mathrm{H}}$. $\alpha$-Spec is localized along the apical basal membrane whereas $\beta$ - and $\beta_{\mathrm{H}-S p e c}$ are localized at basolateral and apical membrane, respectively (88). Cell tension by stretching of wing disc reduces $\mathrm{Crb}$ and apical Spec, resulting in increased Yki activity and organ size. In contrast, compression of wing tissue by overexpression of Crb reduces Yki activity (89). Apical $\beta_{\mathrm{H}-S p e c}$ encoded by karst gene binds to FERM domain proteins such as Ex and Mer to inhibit Yki activity. Thus, spectrin is required as mechnosensor and upstream regulator of Yki in several tissues, including the eye, wing, and oocyte. The link between spectrin and Hippo signaling varies depending on tissues. Basolateral $\beta$-Spec is essential for Yki repression in ovary and intestinal epithelium. However, apical $\alpha$-Spec is dispensable (90). In contrast, loss of $\alpha$-Spec increases organ size and expression levels of Yki target genes in eye and wing discs (91). Interestingly, loss of $\alpha$-Spec causes increased phosphorylation of myosin II light chain (MLC), suggesting that $\alpha$-Spec affects Yki signaling by modulating actomyosin activity in parallel with the Crb-Ex pathway. Spectrin is also essential for YAP regulation in response to cell density in human cells (89).

Epithelial cells in growing organs are subjected to mechanical tension. In normal wing disc development, cell proliferation occurs relatively evenly without distortion of the epithelia, although secreted growth signal such as Decapentaplegic (Dpp)/TGF $\beta$ is locally expressed along the anterior-posterior boundary. Mechanical feedback hypothesis has been proposed to explain such homeostatic regulation of growth in developing organs. In this hypothesis, higher proliferation signaling in fast growing cells are counterbalanced by higher compression and reduced mechanical tension as more cells are produced. A recent study has provided evidence that supports this hypothesis in vivo (92). In normal development, fast growth induced by local mitogenic signals results in a mechanical feedback which activates Hippo signaling by reducing the level of junctional Jub and Wts (Fig. 3). However, when mechanical feedback is blocked by overexpressing Yki target gene ban or depleting Wts, clones of fast-growing cells will lead to increased cell proliferation, consistent with the proposed model.

\section{CONCLUDING REMARKS}

Hippo signaling has emerged as a major conserved mechanism for controlling organ growth. This review is mainly focused on upstream paths that lead to the regulation of Hpo kinase cascade. Hpo signaling is activated by two upstream paths involving $\mathrm{Ft}$ and $\mathrm{Crb}$. Crb function is in part relayed to Hpo through a complex of Ex, Mer, and Kibra and Schip1-Tao1 activity. Functions of Ex, Mer, and Kibra are redundant or independent in different tissues. Thus, regulation of Hippo signaling in cellular and developmental contexts is an 
important issue.

Hippo signaling is also modulated by cytoskeletons. It remains to be studied how actin and spectrin cytoskeleton regulates apical-basal localization and levels of Hippo upstream components. Homeostatic control of growth signaling is critical for organ development. Molecular mechanisms for sensing extracellular environments and transducing cytoskeletal tension to Hippo signaling merit further studies. Roles of microtubules associated with Hippo components are also open questions. Lastly, cross-talks between the Hippo pathway and other signaling networks are important for organ development. Drosophila is an excellent system for genetic dissection of such complex interactions in vivo. Studies in flies and mammals are complementary in providing novel insights into growth regulation.

\section{ACKNOWLEDGEMENTS}

I acknowledge Kyungok Cho, Jean Jung, and Dae-Wook Yang for their valuable comments. I thank Eunjin Yu for improving figures. This study was supported by grants (NRF-2014K1A1 A2042982 and NRF-2017R1A2B3007516) of the National Research Foundation of Korea funded by the Ministry of Education, Science \& Technology, Republic of Korea.

\section{CONFLICTS OF INTEREST}

The authors have no conflicting interests.

\section{REFERENCES}

1. Rieder LE and Larschan EN (2014) Wisdom from the fly. Trends Genet 30, 479-481

2. Xu T, Wang W, Zhang S, Stewart RA and Yu W (1995) Identifying tumor suppressors in genetic mosaics: the Drosophila lats gene encodes a putative protein kinase. Development 121, 1053-1063

3. Justice RW, Zilian O, Woods DF, Noll M and Bryant PJ (1995) The Drosophila tumor suppressor gene warts encodes a homolog of human myotonic dystrophy kinase and is required for the control of cell shape and proliferation. Genes Dev 9, 534-546

4. Tapon N, Harvey KF, Bell DW et al (2002) salvador Promotes both cell cycle exit and apoptosis in Drosophila and is mutated in human cancer cell lines. Cell 110, 67-78

5. Harvey KF, Pfleger CM and Hariharan IK (2003) The Drosophila Mst ortholog, hippo, restricts growth and cell proliferation and promotes apoptosis. Cell 114, 457-467

6. Pantalacci S, Tapon N and Léopold P (2003) The Salvador partner Hippo promotes apoptosis and cell-cycle exit in Drosophila. Nat Cell Biol 5, 921-927

7. Udan RS, Kango-Singh M, Nolo R, Tao C and Halder G (2003) Hippo promotes proliferation arrest and apoptosis in the Salvador/Warts pathway. Nat Cell Biol 5, 914-920

8. Wu S, Huang J, Dong J and Pan D (2003) hippo encodes a
Ste-20 family protein kinase that restricts cell proliferation and promotes apoptosis in conjunction with salvador and warts. Cell 114, 445-456

9. Watt KI, Harvey KF and Gregorevic P (2017) Gregorevic, Regulation of Tissue Growth by the Mammalian Hippo Signaling Pathway. Front Physiol 8, 942

10. Richardson HE and Portela M (2017) Tissue growth and tumorigenesis in Drosophila: cell polarity and the Hippo pathway. Curr Opin Cell Biol 48, 1-9

11. Fulford A, Tapon N and Ribeiro PS (2017) Upstairs, downstairs: spatial regulation of Hippo signalling. Curr Opin Cell Biol 51, 22-32

12. Meng Z, Moroishi T and Guan KL (2016) Guan, Mechanisms of Hippo pathway regulation. Genes Dev 30, $1-17$

13. Watson KL (1995) Drosophila warts-tumor suppressor and member of the myotonic dystrophy protein kinase family. Bioessays 17, 673-676

14. Zheng Y, Wang W, Liu B, Deng H, Uster E and Pan D (2015) Identification of Happyhour/MAP4K as Alternative $\mathrm{Hpo} / \mathrm{Mst}-\mathrm{like}$ Kinases in the Hippo Kinase Cascade. Dev Cell 34, 642-655

15. Huang J, Wu S, Barrera J, Matthews K and Pan D (2005) The Hippo signaling pathway coordinately regulates cell proliferation and apoptosis by inactivating Yorkie, the Drosophila Homolog of YAP. Cell 122, 421-434

16. Thompson BJ and Cohen SM (2006) The Hippo pathway regulates the bantam microRNA to control cell proliferation and apoptosis in Drosophila. Cell 126, 767-774

17. Nolo R, Morrison CM, Tao C, Zhang X and Halder G (2006) The bantam microRNA is a target of the hippo tumor-suppressor pathway. Curr Biol 16, 1895-1904

18. Simpson P, Lawrence PA and Maschat F (1981) Clonal analysis of two wing-scalloping mutants of Drosophila. Dev Biol 84, 206-211

19. Campbell S, Inamdar $M$, Rodrigues V, Raghavan V, Palazzolo M and Chovnick A (1992) The scalloped gene encodes a novel, evolutionarily conserved transcription factor required for sensory organ differentiation in Drosophila. Genes Dev 6, 367-379

20. Halder G, Polaczyk P, Kraus ME et al (1998) The Vestigial and Scalloped proteins act together to directly regulate wing-specific gene expression in Drosophila. Genes Dev 12, 3900-3909

21. Goulev Y, Fauny JD, Gonzalez-Marti B, Flagiello D, Silber $J$ and Zider A (2008) SCALLOPED interacts with YORKIE, the nuclear effector of the hippo tumor-suppressor pathway in Drosophila. Curr Biol 18, 435-441

22. Wu S, Liu Y, Zheng Y, Dong J and Pan D (2008) The TEAD/TEF family protein Scalloped mediates transcriptional output of the Hippo growth-regulatory pathway. Dev Cell 14, 388-398

23. Zhang L, Ren F, Zhang Q, Chen Y, Wang B and Jiang J (2008) The TEAD/TEF family of transcription factor Scalloped mediates Hippo signaling in organ size control. Dev Cell 14, 377-387

24. Meserve JH and Duronio RJ (2015) Scalloped and Yorkie are required for cell cycle re-entry of quiescent cells after tissue damage. Development 142, 2740-2751

25. Yang CH, Axelrod JD and Simon MA (2002) Regulation of 
Frizzled by fat-like cadherins during planar polarity signaling in the Drosophila compound eye. Cell 108, 675-688

26. Silva $\mathrm{E}$, Tsatskis $\mathrm{Y}$, Gardano L, Tapon $\mathrm{N}$ and McNeill $\mathrm{H}$ (2006) The tumor-suppressor gene fat controls tissue growth upstream of expanded in the hippo signaling pathway. Curr Biol 16, 2081-2089

27. Willecke $M$, Hamaratoglu F, Kango-Singh $M$ et al (2006) The fat cadherin acts through the hippo tumor-suppressor pathway to regulate tissue size. Curr Biol 16, 2090-2100

28. Tyler DM and Baker NE (2007) Expanded and fat regulate growth and differentiation in the Drosophila eye through multiple signaling pathways. Dev Biol 305, 187-201

29. Bennett FC and Harvey KF (2006) Fat cadherin modulates organ size in Drosophila via the Salvador/Warts/Hippo signaling pathway. Curr Biol 16, 2101-2110

30. Cho E, Feng Y, Rauskolb C, Maitra S, Fehon R and Irvine KD (2006) Delineation of a Fat tumor suppressor pathway. Nat Genet 38, 1142-1150

31. Vrabioiu AM and Struhl G (2015) Fat/Dachsous Signaling Promotes Drosophila Wing Growth by Regulating the Conformational State of the NDR Kinase Warts. Dev Cell 35, 737-749

32. Misra JR and Irvine KD (2016) Vamana Couples Fat Signaling to the Hippo Pathway. Dev Cell 39, 254-266

33. Feng $Y$ and Irvine KD (2009) Processing and phosphorylation of the Fat receptor. Proc Natl Acad Sci U S A 106, 11989-11994

34. Matakatsu H, Blair SS and Fehon RG (2017) The palmitoyltransferase Approximated promotes growth via the Hippo pathway by palmitoylation of Fat. J Cell Biol 216, 265-277

35. Tepass U, Theres C and Knust E (1990) crumbs encodes an EGF-like protein expressed on apical membranes of Drosophila epithelial cells and required for organization of epithelia. Cell 61, 787-799

36. Pocha SM and Knust E (2013) Complexities of Crumbs function and regulation in tissue morphogenesis. Curr Biol 23, R289-293

37. Chen CL, Gajewski KM, Hamaratoglu F et al (2010) The apical-basal cell polarity determinant Crumbs regulates Hippo signaling in Drosophila. Proc Natl Acad Sci U S A 107, 15810-15815

38. Grzeschik NA, Parsons LM, Allott ML, Harvey KF and Richardson HE (2010) Lgl, aPKC, and Crumbs regulate the Salvador/Warts/Hippo pathway through two distinct mechanisms. Curr Biol 20, 573-581

39. Ling C, Zheng Y, Yin F et al (2010) The apical transmembrane protein Crumbs functions as a tumor suppressor that regulates Hippo signaling by binding to Expanded. Proc Natl Acad Sci U S A 107, 10532-10537

40. Robinson BS, Huang J, Hong Y and Moberg KH (2010) Crumbs regulates Salvador/Warts/Hippo signaling in Drosophila via the FERM-domain protein Expanded. Curr Biol 20, 582-590

41. Margolis B (2017) The Crumbs3 Polarity Protein. Cold Spring Harb Perspect Biol [Epub ahead of print]

42. Ribeiro P, Holder M, Frith D, Snijders AP and Tapon N (2014) Crumbs promotes expanded recognition and degradation by the SCF(Slimb/beta-TrCP) ubiquitin ligase.
Proc Natl Acad Sci U S A 111, E1980-1989

43. Petrilli AM and Fernández-Valle C (2016) Role of Merlin/NF2 inactivation in tumor biology. Oncogene 35, 537-548

44. Lajeunesse DR, McCartney BM and Fehon RG (1998) Structural analysis of Drosophila merlin reveals functional domains important for growth control and subcellular localization. J Cell Biol 141, 1589-1599

45. McCartney BM and Fehon RG (1996) Distinct cellular and subcellular patterns of expression imply distinct functions for the Drosophila homologues of moesin and the neurofibromatosis 2 tumor suppressor, merlin. J Cell Biol 133, 843-852

46. McCartney BM, Kulikauskas RM, Laleunesse DR and Fehon RG (2000) The neurofibromatosis-2 homologue, Merlin, and the tumor suppressor expanded function together in Drosophila to regulate cell proliferation and differentiation. Development 127, 1315-1324

47. Pellock BJ, Buff E, White K and Hariharan IK (2007) The Drosophila tumor suppressors Expanded and Merlin differentially regulate cell cycle exit, apoptosis, and Wingless signaling. Dev Biol 304, 102-115

48. Kremerskothen J, Plaas C, Büther K et al (2003) Characterization of KIBRA, a novel WW domain-containing protein. Biochem Biophys Res Commun 300, 862-867

49. Baumgartner R, Poernbacher I, Buser N, Hafen E and Stocker H (2010) The WW domain protein Kibra acts upstream of Hippo in Drosophila. Dev Cell 18, 309-316

50. Yu J, Zheng Y, Dong J, Klusza S, Deng WM and Pan D (2010) Kibra functions as a tumor suppressor protein that regulates Hippo signaling in conjunction with Merlin and Expanded. Dev Cell 18, 288-299

51. Genevet A, Wehr MC, Brain R, Thompson BJ and Tapon $N$ (2010) Kibra is a regulator of the Salvador/Warts/Hippo signaling network. Dev Cell 18, 300-308

52. Badouel C, Gardano L, Amin N et al (2009) The FERM-domain protein Expanded regulates Hippo pathway activity via direct interactions with the transcriptional activator Yorkie. Dev Cell 16, 411-420

53. MacDougall N, Lad Y, Wilkie GS, Francis-Lang $H$, Sullivan W and Davis I (2001) Merlin, the Drosophila homologue of neurofibromatosis-2, is specifically required in posterior follicle cells for axis formation in the oocyte. Development 128, 665-673

54. Su T, Ludwig MZ, Xu J and Fehon RG (2017) Kibra and Merlin Activate the Hippo Pathway Spatially Distinct from and Independent of Expanded. Dev Cell 40, 478-490 e3

55. Martin PM, Carnaud M, Garcia del Caño G et al (2008) Schwannomin-interacting protein-1 isoform IQCJ-SCHIP-1 is a late component of nodes of Ranvier and axon initial segments. J Neurosci 28, 6111-6117

56. Boggiano JC, Vanderzalm PJ and Fehon RG (2011) Tao-1 phosphorylates Hippo/MST kinases to regulate the Hippo-Salvador-Warts tumor suppressor pathway. Dev Cell 21, 888-895

57. Poon CL, Lin JI, Zhang X and Harvey KF (2011) The sterile 20-like kinase Tao-1 controls tissue growth by regulating the Salvador-Warts-Hippo pathway. Dev Cell 21, 896-906

58. Chung HL, Augustine GJ2 and Choi KW (2016) Drosophila Schip1 Links Expanded and Tao-1 to Regulate 
Hippo Signaling. Dev Cell 36, 511-524

59. Liu T, Rohn JL, Picone R, Kunda P and Baum B (2010) Tao-1 is a negative regulator of microtubule plus-end growth. J Cell Sci 123(Pt 16), 2708-2716

60. Pflanz R, Voigt A, Yakulov T and Jäckle H (2015) Drosophila gene tao- 1 encodes proteins with and without a Ste20 kinase domain that affect cytoskeletal architecture and cell migration differently. Open Biol 5, 140161

61. Poon CL, Mitchell KA, Kondo S, Cheng LY and Harvey KF (2016) The Hippo Pathway Regulates Neuroblasts and Brain Size in Drosophila melanogaster. Curr Biol 26, 1034-1042

62. Chung HL and Choi KW (2016) Schip1, a new upstream regulator of Hippo signaling. Cell Cycle 15, 2097-2098

63. Dewey EB, Taylor DT and Johnston CA (2016) Rolling in the mud: Hippo controls oriented cell division. Cell Cycle 15, 607-608

64. Keder A, Rives-Quinto N, Aerne BL, Franco M, Tapon N and Carmena A (2015) The hippo pathway core cassette regulates asymmetric cell division. Curr Biol 25, 2739-2750

65. Strzyz P (2015) Cell division: Hippo regulates cell division. Nat Rev Mol Cell Biol 16, 702-703

66. Sansores-Garcia L, Bossuyt W, Wada K et al (2011) Modulating F-actin organization induces organ growth by affecting the Hippo pathway. EMBO J 30, 2325-2335

67. Fernández BG, Gaspar P, Brás-Pereira C, Jezowska B, Rebelo SR and Janody F (2011) Actin-Capping Protein and the Hippo pathway regulate F-actin and tissue growth in Drosophila. Development 138, 2337-2346

68. Bosveld F, Guirao B, Wang Z et al (2016) Modulation of junction tension by tumor suppressors and protooncogenes regulates cell-cell contacts. Development 143, 623-634

69. Ko C, Kim YG, Le TP and Choi KW (2016) Twinstar/cofilin is required for regulation of epithelial integrity and tissue growth in Drosophila. Oncogene 35, 5144-5154

70. Janody F and Treisman JE (2006) Actin capping protein alpha maintains vestigial-expressing cells within the Drosophila wing disc epithelium. Development 133, 3349-3357

71. Butcher DT, Alliston T and Weaver VM (2009) A tense situation: forcing tumour progression. Nat Rev Cancer 9, 108-122

72. Dupont S, Morsut L, Aragona $M$ et al (2011) Role of YAP/TAZ in mechanotransduction. Nature 474, 179-183

73. Baumann K (2014) Development. Mechanical forces linked to organ growth. Nat Rev Mol Cell Biol 15, 501

74. Yu FX, Zhao B and Guan KL (2015) Hippo Pathway in Organ Size Control, Tissue Homeostasis, and Cancer. Cell $163,811-828$

75. Das Thakur $M$, Feng $Y$, Jagannathan $R$, Seppa MJ, Skeath JB and Longmore GD (2010) Ajuba LIM proteins are negative regulators of the Hippo signaling pathway. Curr Biol 20, 657-662

76. Jagannathan R, Schimizzi GV, Zhang $K$ et al (2016)
AJUBA LIM Proteins Limit Hippo Activity in Proliferating Cells by Sequestering the Hippo Core Kinase Complex in the Cytosol. Mol Cell Biol 36, 2526-2542

77. Rauskolb C, Sun S, Sun G, Pan Y and Irvine KD (2014) Cytoskeletal tension inhibits Hippo signaling through an Ajuba-Warts complex. Cell 158, 143-156

78. Reddy BV and Irvine KD (2013) Regulation of Hippo signaling by EGFR-MAPK signaling through Ajuba family proteins. Dev Cell 24, 459-471

79. Sun G and Irvine KD (2013) Irvine, Ajuba family proteins link JNK to Hippo signaling. Sci Signal 6, ra81

80. Yonemura S, Wada Y, Watanabe T, Nagafuchi A and Shibata M (2010) alpha-Catenin as a tension transducer that induces adherens junction development. Nat Cell Biol 12, 533-542

81. Maki K, Han SW, Hirano Y, Yonemura S, Hakoshima T and Adachi T (2016) Mechano-adaptive sensory mechanism of alpha-catenin under tension. Sci Rep 6, 24878

82. Trichet L, Sykes C and Plastino J (2008) Relaxing the actin cytoskeleton for adhesion and movement with Ena/VASP. J Cell Biol 181, 19-25

83. Gaspar P, Holder MV, Aerne BL, Janody F and Tapon N (2015) Zyxin antagonizes the FERM protein expanded to couple F-actin and Yorkie-dependent organ growth. Curr Biol 25, 679-689

84. Rauskolb C, Pan G, Reddy BV, Oh H and Irvine KD (2011) Zyxin links fat signaling to the hippo pathway. PLoS Biol 9, e1000624

85. Harvey KF (2015) Growth control: re-examining Zyxin's role in the hippo pathway. Curr Biol 25, R230-231

86. Aragona M, Panciera T, Manfrin A et al (2013) A mechanical checkpoint controls multicellular growth through YAP/TAZ regulation by actin-processing factors. Cell 154, 1047-1059

87. Halder G, Dupont S and Piccolo S (2012) Transduction of mechanical and cytoskeletal cues by YAP and TAZ. Nat Rev Mol Cell Biol 13, 591-600

88. Lee JK, Brandin E, Branton D and Goldstein LS (1997) alpha-Spectrin is required for ovarian follicle monolayer integrity in Drosophila melanogaster. Development 124, 353-362

89. Fletcher GC, Elbediwy A, Khanal I, Ribeiro PS, Tapon N and Thompson BJ (2015) The Spectrin cytoskeleton regulates the Hippo signalling pathway. EMBO J 34, 940-954

90. Wong KK, Li W, An Y et al (2015) beta-Spectrin regulates the hippo signaling pathway and modulates the basal actin network. J Biol Chem 290, 6397-6407

91. Deng $H$, Wang $W, Y u J$, Zheng $Y$, Qing $Y$ and Pan D (2015) Spectrin regulates Hippo signaling by modulating cortical actomyosin activity. Elife 4, e06567

92. Pan Y, Heemskerk I, Ibar C, Shraiman BI and Irvine KD (2016) Differential growth triggers mechanical feedback that elevates Hippo signaling. Proc Natl Acad Sci U S A [Epub ahead of print] 